\title{
ON THE TODA AND KAC-VAN MOERBEKE SYSTEMS
}

\author{
F. GESZTESY, H. HOLDEN, B. SIMON, AND Z. ZHAO
}

Dedicated to Michael J. Moravcsik (1928-1989)

\begin{abstract}
Given a solution of the Toda lattice we explicitly construct a solution of the Kac-van Moerbeke system related to each other by a Miura-type transformation. As an illustration of our method we derive the $N$-soliton solutions of the Kac-van Moerbeke lattice.
\end{abstract}

\section{INTRODUCTION}

We extend our previous work $[11,12]$ on conncections between the KortewegdeVries and modified Korteweg-deVries equation based on Miura's transformation [23] to the Toda lattice and its modified version, the Kac-van Moerbeke system. The Toda and Kac-van Moerbeke equations, in appropriate variables [7], are defined by $[19,22,26,29]$

$$
\begin{aligned}
& \dot{a}(t, n)=a(t, n)[b(t, n)-b(t, n+1)], \\
& \dot{b}(t, n)=2\left[a(t, n-1)^{2}-a(t, n)^{2}\right], \quad(t, n) \in \mathbb{R} \times \mathbb{Z},
\end{aligned}
$$

and

$$
\dot{\alpha}(t, n)=\alpha(t, n)\left[\alpha(t, n+1)^{2}-\alpha(t, n-1)^{2}\right], \quad(t, n) \in \mathbb{R} \times \mathbb{Z},
$$

respectively. Here "•" denotes $d / d t, a, b, \alpha$ are real-valued sequences, and $(a+b)(n):=a(n)+b(n),(a b)(n):=a(n) b(n), n \in \mathbb{Z}$. The analog of Miura's transformation between the two nonlinear lattices now reads as follows. Let $\alpha$ be a solution of the Kac-van Moerbeke system (1.2) and define

$$
\begin{aligned}
& a_{1}(t, n):=\alpha(t, 2 n) \alpha(t, 2 n+1), \\
& a_{2}(t, n):=\alpha(t, 2 n+1) \alpha(t, 2 n+2), \\
& b_{1}(t, n):=-\alpha(t, 2 n-1)^{2}-\alpha(t, 2 n)^{2}, \\
& b_{2}(t, n):=-\alpha(t, 2 n)^{2}-\alpha(t, 2 n+1)^{2}, \quad(t, n) \in \mathbb{R} \times \mathbb{Z} .
\end{aligned}
$$

Then $\left(a_{1}, b_{1}\right)$ and $\left(a_{2}, b_{2}\right)$ solve the Toda lattice equations (1.1). Our main objective in this paper is to reverse this process, i.e., starting with a solution, say $\left(a_{1}, b_{1}\right)$ of $(1.1)$ we shall construct a solution $\alpha$ of (1.2) and another solution $\left(a_{2}, b_{2}\right)$ of $(1.1)$ that are linked to each other by the Miura-type transformations (1.3).

Received by the editors July 22, 1991.

1991 Mathematics Subject Classification. Primary 58F07; Secondary 35Q53.

The second and third authors were partially supported by USNSF Grant DMS-8801918. 
The transformations (1.3), according to a footnote in [24], seem to go back to $M$. Hénon. They have been used in [24] in connection with the scattering problem for the finite Kac-van Moerbeke system [19]. The corresponding semiinfinite system has been studied in [19] (see also [31]). The Kac-van Moerbeke system (1.2) on $\mathbb{Z}$ is treated, e.g., in [22, 26, 29] and, due to its applications in modeling Langmuir oscillations in plasmas, is also called the Langmuir lattice. For studies of the $2 N$-periodic system (1.2) (i.e., $\alpha(t, n+2 N)=\alpha(t, n)$ ) we refer, e.g., to $[1,20,21,32]$.

In $\S 2$ we present our main arguments concerning the construction of solutions $\alpha$ of (1.2) given a solution $\left(a_{1}, b_{1}\right)$ of (1.1) related to each other via (1.3). As a technical tool we employ certain results on positive, weak solutions of second order finite difference operators which are summarized in Appendix A. In $\S 3$ we transfer the class of $N$-soliton solutions of the Toda lattice (1.1) to the Kac-van Moerbeke system (1.2).

Finally we mention that our methods are not confined to $(1+1)$-dimensional systems such as the (modified) Korteweg-deVries equation, Gelfand-Dikii and Drinfeld-Sokolov hierarchies, and Toda systems, but also apply to the (modified) Kadomtsev-Petviashvili equation [9].

\section{Connections between the Toda and Kac-van Moerbeke systems}

In this section we extend our previous treatment of the Korteweg-deVries and modified Korteweg-deVries equations [11], [12], to the Toda lattice and its modified version, the Kac-van Moerbeke system.

In $l^{\infty}(\mathbb{Z})$ we introduce shift operators $S^{ \pm}$by

$$
\left(S^{ \pm} f\right)(n):=f(n \pm 1), \quad n \in \mathbb{Z}, f=\{f(n)\}_{n \in \mathbf{Z}} \in l^{\infty}(\mathbb{Z}),
$$

and also use the notation

$$
f^{ \pm}:=S^{ \pm} f
$$

Assuming hypothesis

$$
\begin{array}{r}
a(t)=\{a(t, n)\}_{n \in \mathbb{Z}} \in l_{\mathbb{R}(\mathbb{Z}), \quad} \quad b(t)=\{b(t, n)\}_{n \in \mathbf{Z}} \in l_{\mathbb{R}}^{\infty}(\mathbb{Z}), \\
t \in \mathbb{R}, a(\cdot, n), b(\cdot, n) \in C^{\infty}(\mathbb{R}), n \in \mathbb{Z},
\end{array}
$$

the Toda lattice (TL), in Flaschka's variables [7], reads

$$
\operatorname{TL}(a, b):=\left(\dot{a}-a\left[b-b^{+}\right], \dot{b}-2\left[\left(a^{-}\right)^{2}-a^{2}\right]\right)=0,
$$

where "*" denotes $d / d t$ and $l_{\mathbf{R}}^{\infty}(\mathbb{Z})$ denotes the algebra of real-valued sequences in $l^{\infty}(\mathbb{Z})$.

Assuming hypothesis

$$
\alpha(t)=\{\alpha(t, n)\}_{n \in \mathbf{Z}} \in l_{\mathbf{R}}^{\infty}(\mathbb{Z}), \quad t \in \mathbb{R}, \alpha(\cdot, n) \in C^{\infty}(\mathbb{R}), n \in \mathbb{Z},
$$

the corresponding modified Toda lattice, the Kac-van Moerbeke (KM) lattice is then defined by

$$
\operatorname{KM}(\alpha):=\dot{\alpha}-\alpha\left[\left(\alpha^{+}\right)^{2}-\left(\alpha^{-}\right)^{2}\right]=0 .
$$

The connection between (2.3) and (2.4) is the following: Suppose $\alpha$ satisfies (H.2.2) and (2.4) and define

$$
\phi(t, n):=\alpha(t, 2 n), \quad \psi(t, n):=\alpha(t, 2 n+1), \quad(t, n) \in \mathbb{R} \times \mathbb{Z},
$$


and

$$
\begin{array}{lll}
a_{1}(t):=\phi(t) \psi(t), & b_{1}(t):=-\phi(t)^{2}-\psi^{-}(t)^{2}, & t \in \mathbb{R}, \\
a_{2}(t):=\phi^{+}(t) \psi(t), & b_{2}(t):=-\phi(t)^{2}-\psi(t)^{2}, & t \in \mathbb{R} .
\end{array}
$$

Then $\left(a_{j}, b_{j}\right), j=1,2$, satisfy (H.2.1) and (2.3). In particular,

$$
\mathrm{KM}(\alpha)=0 \Rightarrow \operatorname{TL}\left(a_{j}, b_{j}\right)=0, \quad j=1,2
$$

$[19,24]$ (see also [1, 26, 29]). Thus (2.6), (2.7) play a role analogous to Miura's transformation [23] in the (modified) Korteweg-deVries context. We also note that the transform from $\left(a_{1}, b_{1}\right)$ to $\left(a_{2}, b_{2}\right)$ is simply given by the replacement

$$
\alpha \rightarrow S^{+} \alpha
$$

in (1.3). Our main objective in this paper is to reverse the implication in (2.8), i.e., given a solution $\left(a_{1}, b_{1}\right)$ of (2.3), construct solutions $\alpha$ of (2.4) and $\left(a_{2}, b_{2}\right)$ of $(2.3)$ related to each other by $(2.6)-(2.8)$.

We start by recalling the Lax pairs associated with the TL and KM equations and refer to [5] for relevant Hilbert space methods. Assume (H.2.1) and define on $l^{2}(\mathbb{Z})$

$$
\begin{gathered}
H(t):=a(t) S^{+}+a^{-}(t) S^{-}-b(t), \quad t \in \mathbb{R}, \\
B_{H}(t):=a(t) S^{+}-a^{-}(t) S^{-}, \quad t \in \mathbb{R}, \\
A(t):=\psi(t) S^{+}+\phi(t), \quad A(t)^{*}=\psi^{-}(t) S^{-}+\phi(t), \quad t \in \mathbb{R},
\end{gathered}
$$

and on $l^{2}(\mathbb{Z}) \otimes \mathbb{C}^{2}$

$$
Q(t):=\left(\begin{array}{cc}
0 & A(t)^{*} \\
A(t) & 0
\end{array}\right), \quad t \in \mathbb{R}
$$

$$
B_{Q}(t):=\left(\begin{array}{cc}
B_{A^{*} A}(t) & 0 \\
0 & B_{A A^{*}}(t)
\end{array}\right)=B_{A^{*} A}(t) \oplus B_{A A^{*}}(t), \quad t \in \mathbb{R},
$$

where $\phi$ and $\psi$ are defined as in (2.5). Then

$$
\dot{H}-\left[B_{H}, H\right]=0 \quad \text { iff } \quad \operatorname{TL}(a, b)=0
$$

and

$$
\dot{Q}-\left[B_{Q}, Q\right]=0 \quad \text { iff } \quad \mathrm{KM}(\alpha)=0
$$

implying

Theorem 2.3 [7, 19] (see also [24, 26, 29]). (i) Suppose (a, b) satisfies (H.2.1) and $\operatorname{TL}(a, b)=0$. Then there exists a family of unitary operators $U(t), t \in$ $\mathbb{R}, U(0)=1$ in $l^{2}(\mathbb{Z})$ such that

$$
U(t)^{-1} H(t) U(t)=H(0), \quad t \in \mathbb{R} .
$$

(ii) Suppose $\alpha$ satisfies (H.2.2) and $\mathrm{KM}(\alpha)=0$. Then there exists a family of unitary operators $W(t), t \in \mathbb{R}, W(0)=1$ in $l^{2}(\mathbb{Z}) \otimes \mathbb{C}^{2}$ such that

$$
W(t)^{-1} Q(t) W(t)=Q(0), \quad t \in \mathbb{R} \text {. }
$$


Clearly this theorem extends to unbounded sequences $a, b, \alpha$ as long as appropriate domain considerations for $H(t)$ and $Q(t)$ are taken into account.

Next we recall the definition of the Wronskian determinant associated with (2.10),

$$
W(f, g)(n):=a(n)[f(n) g(n+1)-f(n+1) g(n)],
$$

and define weak solutions $f$ of $H f=z f, z \in \mathbb{C}$, by

$$
((H-\bar{z}) g, f):=\sum_{n \in \operatorname{supp}(g)} \overline{((H-\bar{z}) g)(n)} f(n)=0, g \in l_{0}(\mathbb{Z}),
$$

where $l_{0}(\mathbb{Z})$ denotes the set of complex-valued sequences $g=\{g(n)\}_{n \in \mathbf{Z}}$ with only finitely many $g(n) \neq 0$.

Lemma 2.4. Suppose $(a, b)$ satisfies (H.2.1) and $\operatorname{TL}(a, b)=0$ and let $f_{0}(\lambda)$ be a real-valued weak solution of $H(0) f_{0}(\lambda)=\lambda f_{0}(\lambda), \lambda \in \mathbb{R}$. Then

$$
H(t) f(t, \lambda)=\lambda f(t, \lambda), \quad t \in \mathbb{R}, \lambda \in \mathbb{R},
$$

has a unique real-valued weak solution $f(t, \lambda)$ with $f(\cdot, \lambda, n) \in C^{\infty}(\mathbb{R}), \lambda \in$ $\mathbb{R}, n \in \mathbb{Z}$, satisfying the time evolution

$$
\begin{aligned}
\dot{f}(t, \lambda) & =\left(B_{H}(t) f\right)(t, \lambda)=a(t) f^{+}(t, \lambda)-a^{-}(t) f^{-}(t, \lambda) \\
& =-2 a^{-}(t) f^{-}(t, \lambda)+[\lambda+b(t)] f(t, \lambda) \\
& =2 a(t) f^{+}(t, \lambda)-[\lambda+b(t)] f(t, \lambda), \quad t \in \mathbb{R}, \lambda \in \mathbb{R},
\end{aligned}
$$

in the weak sense with

$$
f(0, \lambda)=f_{0}(\lambda), \quad \lambda \in \mathbb{R} .
$$

(Clearly $f$ is smooth w.r.t. $\lambda$ if $f_{0}$ is.) Moreover, if $f(t, \lambda), g(t, \lambda)$ are two weak solutions of (2.21) and (2.22) with initial values $f_{0}(\lambda), g_{0}(\lambda)$ respectively, then

$$
W(f(t, \lambda), g(t, \lambda))(n)=W\left(f_{0}(\lambda), g_{0}(\lambda)\right)(n), \quad \lambda \in \mathbb{R},
$$

is independent of $(t, n) \in \mathbb{R} \times \mathbb{Z}$.

Proof. A direct calculation shows that

$$
F(t, \lambda):=\dot{f}(t, \lambda)-2 a(t) f^{+}(t, \lambda)+[\lambda+b(t)] f(t, \lambda), \quad(t, \lambda) \in \mathbb{R}^{2}
$$

satisfies

$$
H(t) F(t, \lambda)=\lambda F(t, \lambda), \quad(t, \lambda) \in \mathbb{R}^{2},
$$

in the weak sense whenever $f(t, \lambda)$ satisfies $H(t) f(t, \lambda)=\lambda f(t, \lambda)$ in the weak sense. The requirement $(2.22)$, i.e.,

$$
F(t, \lambda)=0,(t, \lambda) \in \mathbb{R}^{2},
$$

using the equation

$$
\begin{aligned}
f(t, \lambda, n)= & A(t, \lambda) s^{0}(t, \lambda, n)+B(t, \lambda) c^{0}(t, \lambda, n) \\
& -\sum_{m=0}^{n} a(t, 0)^{-1}\left[c^{0}(t, \lambda, n) s^{0}(t, \lambda, m)-c^{0}(t, \lambda, m) s^{0}(t, \lambda, n)\right] \\
& \cdot b(t, m) f(t, \lambda, m), \quad(t, \lambda, n) \in \mathbb{R}^{2} \times \mathbb{Z},
\end{aligned}
$$


where $s^{0}, c^{0}$ satisfy $(2.21)$ with $b(t) \equiv 0$ and (2.29)

$$
s^{0}(t, \lambda, 0)=c^{0}(t, \lambda, 1)=0, \quad s^{0}(t, \lambda, 1)=c^{0}(t, \lambda, 0)=1, \quad(t, \lambda) \in \mathbb{R}^{2},
$$

is then equivalent to the linear first order system

$$
\begin{array}{r}
\left(\begin{array}{c}
\dot{A} \\
\dot{B}
\end{array}\right)=\left(\begin{array}{cc}
-2 b_{0}+b_{1}+\lambda & a_{0}^{-1}\left[2 \lambda b_{0}-2 a_{-1}^{2}\right] \\
2 a_{0} & b_{0}-\lambda
\end{array}\right)\left(\begin{array}{l}
A \\
B
\end{array}\right), \\
a_{0}(\cdot):=a(\cdot, 0), b_{0}(\cdot):=b(\cdot, 0) .
\end{array}
$$

Since

$$
\begin{aligned}
& f(t, \lambda, 0)=B(t, \lambda), \\
& f(t, \lambda, 1)=A(t, \lambda)+a_{0}(t)^{-1} b_{0}(t) B(t, \lambda), \quad(t, \lambda) \in \mathbb{R}^{2},
\end{aligned}
$$

an appropriate choice of $A(0, \lambda), B(0, \lambda)$ will indeed guarantee (2.22) and (2.23). The equation (2.24) follows by a straightforward computation of $\partial_{t} W(f(t, \lambda), g(t, \lambda))=0,(t, \lambda) \in \mathbb{R}^{2}$.

Since the existence of positive weak solutions of $H(t) f(t)=0$ will play a crucial role in Theorem 2.9 we introduce hypothesis

$$
\begin{aligned}
& (a(t), b(t)) \in l_{\mathbb{R}}^{\infty}(\mathbb{Z}) \times l_{\mathbb{R}}^{\infty}(\mathbb{Z}), \quad t \in \mathbb{R}, \\
& 0>a(\cdot, n) \in C^{\infty}(\mathbb{R}), \quad 0>b(\cdot, n) \in C^{\infty}(\mathbb{R}), \quad n \in \mathbb{Z}, \\
& {[b(0, n) / a(0, n)]=o(|n|) \quad \text { as } n \rightarrow \pm \infty}
\end{aligned}
$$

and state

Lemma 2.6. Assume (H.2.5) and $\operatorname{TL}(a, b)=0$, and let $f_{0}>0$ be a weak, positive solution of $H(0) f_{0}=0$. Then, if $f(t)$ is the unique weak solution of

$$
H(t) f(t)=0, \quad \dot{f}(t)=B_{H}(t) f(t), \quad t \in \mathbb{R}, f(0)=f_{0}>0,
$$

given by Lemma 2.4, we get

$$
f(t)>0 \text { for all } t \in \mathbb{R} .
$$

Proof. Equation (2.22a) with $\lambda=0$ and the transformation

$$
g(t, n)=f(t, n) e^{-\int_{0}^{t} d s b(s, n)}, \quad(t, n) \in \mathbb{R} \times \mathbb{Z},
$$

yields

$$
\begin{aligned}
\dot{g}(t, n) & =-2 a(t, n-1) \exp \left\{\int_{0}^{t} d s[b(s, n-1)-b(s, n)]\right\} g(t, n-1) \\
& :=A(t, n-1) g(t, n-1), \quad(t, n) \in \mathbb{R} \times \mathbb{Z},
\end{aligned}
$$

or

$$
g(t, n)=f_{0}(n)+\int_{0}^{t} d s A(s, n-1) g(s, n-1), \quad(t, n) \in \mathbb{R} \times \mathbb{Z} .
$$

Next suppose $t \geq 0$. Then, since $A(t, n)>0, f_{0}(n)>0$, iterating (2.36) yields a positive function $g(t, n)>0$ (implying $f(t, n)>0$ ) whenever the 
iteration procedure converges absolutely. (In that case it is easily verified that the iterations indeed provide a solution of (2.35).) The $m$ th iteration yields (2.37)

$$
\begin{gathered}
\int_{0}^{t} d t_{1} A\left(t_{1}, n-1\right) \int_{0}^{t_{2}} d t_{2} A\left(t_{2}, n-2\right) \cdots \int_{0}^{t_{m-1}} d t_{m} A\left(t_{m}, n-m\right) f_{0}(n-m) \\
\leq C(t)^{m} t^{m}(m !)^{-1} f_{0}(n-m), \quad t \geq 0, \quad n \in \mathbb{Z}, \quad m \in \mathbb{N}_{0},
\end{gathered}
$$

where we used

$$
\sup _{0 \leq s \leq t}\|A(s)\|_{\infty}=C(t), \quad t \geq 0 .
$$

It remains to bound (2.37). First we note that for some $\gamma>0$

$$
f_{0}(k) \leq \gamma\left\{\begin{array}{rlr}
b(0,0) b(0,1) \cdots b(0, k-1) & \\
\quad \times[a(0,0) a(0,1) \cdots a(0, k-1)]^{-1} f_{0}(0), & & k>0 \\
b(0, k) b(0, k-1) \cdots b(0,0) & \\
\quad \times[a(0, k) a(0, k-1) \cdots a(0,0)]^{-1} f_{0}(0), & & k<0 .
\end{array}\right.
$$

This follows from (A.9)-(A.11) since $f_{0}=\alpha u_{-}+\beta u_{+}$for some $\alpha, \beta \geq 0$ and the fact that (2.39) holds for $u_{ \pm}$. Indeed, for $k>0$,

$$
\begin{aligned}
u_{+}(k) & =[-a(0,0)] \cdots[-a(0, k-1)] c_{1,+} \cdots c_{k,+} \\
& \leq b(0,0) \cdots b(0, k-1)[a(0,0) \cdots a(0, k-1)]^{-1}, \\
u_{-}(k) & =[-a(0,0)] \cdots[-a(0, k-1)] c_{0,-} \cdots c_{k-1,-} \\
& \leq b(0,0) \cdots b(0, k-1)[a(0,0) \cdots a(0, k-1)]^{-1}
\end{aligned}
$$

by (A.12) and (A.13). Similarly one treats the case $k<0$. Thus we get

$$
\begin{aligned}
C(t)^{m} t^{m}(m !)^{-1} f_{0}(n-m) \leq \gamma f_{0}(0) C(t)^{m} t^{m}(m !)^{-1} & \\
& \cdot b(0, n-m) \cdots b(0, n-1)[a(0, n-m) \cdots a(0, n-1)]^{-1} \\
:= & \beta_{m}(t, n), \quad t \geq 0, \quad m \in \mathbb{N}_{0}, \quad n \in \mathbb{Z} .
\end{aligned}
$$

Since by hypothesis (H.2.5),

$$
\begin{aligned}
\frac{\beta_{m+1}(t, n)}{\beta_{m}(t, n)} & =t C(t) \frac{b(0, n-m-1)}{a(0, n-m-1)} \frac{1}{m+1} \\
& =t C(t) o(1) \quad \text { as } m \rightarrow \infty,
\end{aligned}
$$

the iterations converge absolutely for $t \geq 0$. For $t \leq 0$ one uses $(2.22 \mathrm{~b})$ with $\lambda=0$ and proceeds analogously.

Remark 2.7. The simple example

$$
\begin{aligned}
& -a(0, n)=\left\{\begin{array}{ll}
(n+1)^{-1}, & n \geq 1, \\
1, & n \leq 0,
\end{array} \quad-b(0, n)= \begin{cases}1+n^{-2}, & n \geq 1, \\
2, & n \leq 0,\end{cases} \right.
\end{aligned}
$$

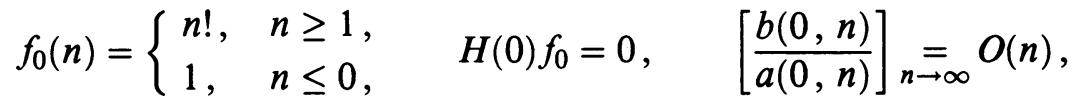

shows that our condition $o(|n|)$ in (H.2.5) cannot be weakened for the iterations (2.37) to converge for all $t \geq 0$. 
Remark 2.8. The connection between Flaschka's and Toda's original variables reads

$$
\begin{aligned}
a(t, n) & :=\left(\epsilon_{n} / 2\right) e^{-[Q(t, n+1)-Q(t, n)] / 2}, \quad b(t, n)=\dot{Q}(t, n) / 2, \\
& \epsilon_{n}= \pm 1, \quad(t, n) \in \mathbb{R} \times \mathbb{Z}, \\
\ddot{Q} & =e^{-\left(Q-Q^{-}\right)}-e^{-\left(Q^{+}-Q\right)},
\end{aligned}
$$

and similarly for the Kac-van Moerbeke system

$$
\begin{aligned}
& \alpha(t, n):=\epsilon_{n}(1 / 2) e^{-R(t, n) / 2}, \quad \epsilon_{n}= \pm 1,(t, n) \in \mathbb{R} \times \mathbb{Z}, \\
& \dot{R}=\left[e^{-R^{-}}-e^{-R^{+}}\right] / 2 .
\end{aligned}
$$

We also note that $T(a, b)=0$ is invariant under

$$
a(t, n) \rightarrow \epsilon_{n} a(t, n), \quad \epsilon_{n}= \pm 1, \quad b(t, n) \rightarrow b(t, n)+c, \quad c \in \mathbb{R}
$$

(independent of $(t, n)$ ), and $\operatorname{KM}(\alpha)=0$ is invariant w.r.t.

$$
\alpha(t, n) \rightarrow \epsilon_{n} \alpha(t, n), \quad \epsilon_{n}= \pm 1 .
$$

These facts motivate our hypothesis $a<0$ in Lemma 2.6. The assumption $b<0$ is then necessary for the existence of $f_{0}>0$ in Lemma 2.6.

Now we are in position to reverse the implication in (2.8).

Theorem 2.9. Assume $\left(a_{1}, b_{1}\right)$ satisfies (H.2.5) and $\operatorname{TL}\left(a_{1}, b_{1}\right)=0$. Suppose $H_{1}(0) \geq 0$ and let $0<u_{1, \pm}(\cdot, n) \in C^{\infty}(\mathbb{R}), n \in \mathbb{Z}$, be positive weak solutions of

$$
H_{1}(t) u_{1, \pm}(t)=0, \quad \dot{u}_{1, \pm}(t)=B_{H_{1}}(t) u_{1, \pm}(t), \quad t \in \mathbb{R}
$$

Define

$$
\begin{aligned}
& u_{1, \sigma}(t, n):=2^{-1}[1-\sigma(t)] u_{1,-}(t, n)+2^{-1}[1+\sigma(t)] u_{1,+}(t, n), \\
& \phi_{\sigma}(t, n):=-\left[-a_{1}(t, n) u_{1, \sigma}(t, n+1) / u_{1, \sigma}(t, n)\right]^{1 / 2}, \\
& \psi_{\sigma}(t, n):=\left[-a_{1}(t, n) u_{1, \sigma}(t, n) / u_{1, \sigma}(t, n+1)\right]^{1 / 2} \text {, } \\
& \alpha_{\sigma}(t, n):= \begin{cases}\phi_{\sigma}(t, m), & n=2 m, \\
\psi_{\sigma}(t, m), & n=2 m+1,\end{cases} \\
& a_{2, \sigma}(t, n):=\phi_{\sigma}(t, n+1) \psi_{\sigma}(t, n) \text {, } \\
& b_{2, \sigma}(t, n):=-\phi_{\sigma}(t, n)^{2}-\psi_{\sigma}(t, n)^{2}, \quad(t, n) \in \mathbb{R} \times \mathbb{Z},
\end{aligned}
$$

where $\sigma: \mathbb{R} \rightarrow[-1,1], \sigma \in C^{\infty}(\mathbb{R})$. Then $\alpha_{\sigma}(t) \in l_{\mathbb{R}}^{\infty}(\mathbb{Z}),\left(a_{2},{ }_{\sigma}(t), b_{2, \sigma}(t)\right)$ $\in l_{\mathbf{R}}^{\infty}(\mathbb{Z}) \times l_{\mathbf{R}}^{\infty}(\mathbb{Z}), t \in \mathbb{R}$, and

(2.54) $K M\left(\alpha_{\sigma}\right)=0, T L\left(a_{2, \sigma}, b_{2, \sigma}\right)=0 \quad$ iff $\quad \dot{\sigma}=0$ or $W\left(u_{1,-}, u_{1,+}\right)=0$.

Proof. Since

$$
-b_{1}(t, n)=\phi_{\sigma}(t, n)^{2}+\psi_{\sigma}(t, n-1)^{2}, \quad(t, n) \in \mathbb{R} \times \mathbb{Z},
$$

and $-b_{1}(t) \in l_{\mathbb{R}}^{\infty}(\mathbb{Z}), t \in \mathbb{R}$, the boundedness assertions for $\alpha_{\sigma}, a_{2, \sigma}, b_{2, \sigma}$ hold. Moreover, one explicitly computes

$$
\begin{array}{r}
\mathrm{KM}\left(\alpha_{\sigma}\right)(t, n)=-4^{-1} \dot{\sigma}(t) \phi_{\sigma}(t, m)^{-1} u_{1, \sigma}(t, m)^{-2} W\left(u_{1,-}, u_{1,+}\right), \\
t \in \mathbb{R}, n=2 m,
\end{array}
$$




$$
\begin{array}{r}
\mathrm{KM}\left(\alpha_{\sigma}\right)(t, n)=4^{-1} \dot{\sigma}(t) \psi_{\sigma}(t, m)^{-1} u_{1, \sigma}(t, m+1)^{-2} W\left(u_{1,-}, u_{1,+}\right), \\
t \in \mathbb{R}, \quad n=2 m+1,
\end{array}
$$

and similarly

$$
\begin{aligned}
& \operatorname{TL}\left(a_{2, \sigma}, b_{2, \sigma}\right)(n)=4^{-1} \dot{\sigma}(t) W\left(u_{1,-}, u_{1,+}\right) \\
& \cdot\left(u_{1, \sigma}(n+1)^{-2}\left[-\frac{\psi_{\sigma}(n)}{\phi_{\sigma}(n+1)}+\frac{\phi_{\sigma}(n+1)}{\psi_{\sigma}(n)}\right], 2\left[u_{1, \sigma}(n)^{-2}-u_{1, \sigma}(n+1)^{-2}\right]\right), \\
& t \in \mathbb{R}, n \in \mathbb{Z} .
\end{aligned}
$$

The definitions (2.50)-(2.52) are suggested by $H_{1} f_{1}=A^{*} A f_{1}=0$, i.e., by $A f_{1}=0$ (yielding $\psi f_{1}^{+}+\phi f_{1}=0$, cf. (2.12)) and by the requirements $a_{1}=\phi \psi$ (cf. (2.7)) and (2.5).

In order to interpret Theorem 2.19 we recall some notions from Appendix A: $H_{1}(0) \geq 0$ is called critical iff $H_{1}(0) f=0$ has a unique positive weak solution $u_{1,0}>0$ with $u_{1,0}(0)=1$, and $H_{1}(0) \geq 0$ is called subcritical iff $H_{1}(0) f=0$ has two linearly independent positive weak solutions $u_{1, \pm}>0$. Since by Theorem A.5, $H_{1}(0) \geq 0$ has at least one positive weak solution $u_{1}>0$ of $H_{1}(0) f=0$, this case distinction is exhaustive, i.e., either $H_{1}(0) \geq 0$ is critical or subcritical.

Combining Lemmas 2.4 and 2.6 we get

Lemma 2.10. Let $\left(a_{1}, b_{1}\right)$ satisfy (H.2.5) and $\operatorname{TL}\left(a_{1}, b_{1}\right)=0$ and assume that $H_{1}(0) \geq 0$. Then $H_{1}(t)$ is (sub)critical for some $t \in \mathbb{R}$ iff $H_{1}(t)$ is (sub)critical for all $t \in \mathbb{R}$.

Remark 2.11. Given a solution $\left(a_{1}, b_{1}\right)$ of $\operatorname{TL}\left(a_{1}, b_{1}\right)=0$, Theorem 2.9 yields a unique solution, say $\alpha_{0}$ of $\mathrm{KM}\left(\alpha_{0}\right)=0$ and of $(2.7)$ iff $H_{1}(0)$ is critical. In this case we can dispense with the condition that $u_{1,0}$ satisfies $\dot{u}_{1,0}=B_{H_{1}} u_{1,0}$ since by Lemma 2.4 a certain $t$-dependent multiple of $u_{1,0}$ certainly will satisfy it. (This $t$-dependent factor, however, drops out in the definitions (2.50)-(2.52) and hence is irrelevant.) Otherwise, i.e., iff $H_{1}(0)$ is subcritical, we get a oneparameter family of solutions $\alpha_{\sigma}$ of $\operatorname{KM}\left(\alpha_{\sigma}\right)=0$ and (2.7) indexed by $\sigma \in$ $[-1,1]$.

Remark 2.12. The "if part" of Theorem 2.9 essentially has been established by [1] (see also [29]). It is our novel "only if part" in Theorem 2.9 that yields a uniqueness or nonuniqueness result for $\alpha$ depending on whether $H_{1}(0)$ is critical or subcritical.

Remark 2.13. Given $\alpha_{\sigma}$ in (2.52), $H_{1}(t)$ is recovered from $\alpha_{\sigma}$ via

$$
\begin{aligned}
H_{1}(t) & =A_{\sigma}^{*}(t) A_{\sigma}(t), \quad A_{\sigma}(t)=\psi_{\sigma}(t) S^{+}+\phi_{\sigma}(t), \\
\phi_{\sigma}(t, n) & =\alpha(t, 2 n), \quad \psi_{\sigma}(t, n)=\alpha_{\sigma}(t, 2 n+1), \quad(t, n) \in \mathbb{R} \times \mathbb{Z} .
\end{aligned}
$$

Moreover, $H_{1} f_{1}=\lambda f_{1}$ in the weak sense reads

$$
\begin{aligned}
& a_{1}(t, n) f_{1}(t, n+1)+a_{1}(t, n-1) f_{1}(t, n-1)-b_{1}(t, n) f_{1}(t, n)=\lambda f_{1}(t, n), \\
& a_{1}(t)=\phi_{\sigma}(t) \psi_{\sigma}(t), \quad b_{1}(t)=-\phi_{\sigma}(t)^{2}-\psi_{\sigma}^{-}(t)^{2}, \quad(t, n) \in \mathbb{R}^{2}, \sigma \in[-1,1] .
\end{aligned}
$$


Similarly, $H_{2, \sigma}(t):=A_{\sigma}(t) A_{\sigma}(t)^{*}$ implies for weak solutions $f_{2}$ of $H_{2, \sigma} f_{2}=$ $\lambda f_{2}$ that

$$
\begin{aligned}
& a_{2, \sigma}(t, n) f_{2}(t, n+1)+a_{2, \sigma}(t, n-1) f_{2}(t, n-1)-b_{2, \sigma}(t) f_{2}(t, n)=\lambda f_{2}(t, n), \\
& a_{2, \sigma}(t)=\phi_{\sigma}^{+}(t) \psi_{\sigma}(t), b_{2, \sigma}(t)=-\phi_{\sigma}(t)^{2}-\psi_{\sigma}(t)^{2}, \quad(t, n) \in \mathbb{R}^{2}, \sigma \in[-1,1] .
\end{aligned}
$$

\section{Soliton SOlutions for the KaC-VAN MoERbeKe System}

In this section we illustrate the methods of $\S 2$ by deriving the soliton solutions of the Kac-van Moerbeke system given the soliton solutions of the Toda lattice.

First we inductively construct the solitions of the Toda lattice using commutation methods $[4,6,30]$. Consider

$$
H_{0}^{0}\left(z_{1}\right):=-(1 / 2)\left(S^{+}+S^{-}\right)+\Omega_{1}, \quad \Omega_{1}=\left(z_{1}+z_{1}^{-1}\right) / 2,0<z_{1}<1,
$$

with spectrum

$$
\sigma\left(H_{0}^{0}\left(z_{1}\right)\right)=\left[-1+\Omega_{1}, 1+\Omega_{1}\right] .
$$

Writing

$$
H_{0}^{0}\left(z_{1}\right)=A\left(z_{1}\right)^{*} A\left(z_{1}\right),
$$

with

$$
A\left(z_{1}\right):=\psi_{1}\left(z_{1}\right) S_{+}+\phi_{1}\left(z_{1}\right)
$$

we get

$$
-1 / 2=\psi_{1}\left(z_{1}, n\right) \phi_{1}\left(z_{1}, n\right), \quad n \in \mathbb{Z} .
$$

We have

$$
H_{0}^{0}\left(z_{1}\right) u_{0, \pm}\left(z_{1}\right)=0
$$

where

$$
u_{0, \pm}\left(z_{1}, n\right)=z_{1}^{ \pm n}, \quad n \in \mathbb{Z},
$$

and $H_{0}^{0}\left(z_{1}\right)$ is subcritical [14]. Define

$$
u_{0, \mu_{1}}\left(z_{1}\right):=u_{0,-}\left(z_{1}\right)+\mu_{1} u_{0,+}\left(z_{1}\right), \quad \mu_{1}>0 .
$$

Then $H_{0}^{0}\left(z_{1}\right) u_{0, \mu_{1}}\left(z_{1}\right)=0$ implies

$$
A\left(z_{1}\right) u_{0, \mu_{1}}\left(z_{1}\right)=0
$$

and (3.5), (3.8), and (3.9) yield

$$
\begin{aligned}
& \psi_{1}\left(z_{1}, n\right)=\left[\frac{z_{1}^{-n}+\mu_{1} z_{1}^{n}}{2\left(z_{1}^{-n-1}+\mu_{1} z_{1}^{n+1}\right)}\right]^{1 / 2}, \quad n \in \mathbb{Z}, \\
& \phi_{1}\left(z_{1}, n\right)=-\left[\frac{z_{1}^{-n-1}+\mu_{1} z_{1}^{n+1}}{2\left(z_{1}^{-n}+\mu_{1} z_{1}^{n}\right)}\right]^{1 / 2}, \quad n \in \mathbb{Z} .
\end{aligned}
$$

(The signs of $\psi_{1}$ and $\phi_{1}$ are not determined except for the fact that they must be chosen opposite of each other.) Next define

$$
H_{1}\left(z_{1}\right)=A\left(z_{1}\right) A\left(z_{1}\right)^{*}:=a_{1}\left(z_{1}\right) S^{+}+a_{1}^{-}\left(z_{1}\right) S^{-}-b_{1}\left(z_{1}\right) .
$$


Then

$$
\begin{aligned}
& a_{1}\left(z_{1}, n\right)=\phi_{1}\left(z_{1}, n+1\right) \psi_{1}\left(z_{1}, n\right), \\
& b_{1}\left(z_{1}, n\right)=-\phi_{1}\left(z_{1}, n\right)^{2}-\psi_{1}\left(z_{1}, n\right)^{2}, \quad n \in \mathbb{Z},
\end{aligned}
$$

yields

$$
\begin{aligned}
& a_{1}\left(z_{1}, n\right)=-\frac{\left(z_{1}^{-n-2}+\mu_{1} z_{1}^{n+2}\right)^{1 / 2}\left(z_{1}^{-n}+\mu_{1} z_{1}^{n}\right)^{1 / 2}}{2\left(z_{1}^{-n-1}+\mu_{1} z_{1}^{n+1}\right)}, \\
& b_{1}\left(z_{1}, n\right)=-\frac{1}{2}\left[\frac{z_{1}^{-n-1}+\mu_{1} z_{1}^{n+1}}{z_{1}^{-n}+\mu_{1} z_{1}^{n}}+\frac{z_{1}^{-n}+\mu_{1} z_{1}^{n}}{z_{1}^{-n-1}+\mu_{1} z_{1}^{n+1}}\right], \quad n \in \mathbb{Z} .
\end{aligned}
$$

Moreover, the operator $H_{1}\left(z_{1}\right)$ is critical with spectrum

$$
\sigma\left(H_{1}\left(z_{1}\right)\right)=\{0\} \cup\left[-1+\Omega_{1}, 1+\Omega_{1}\right]
$$

since the equation

$$
H_{1}\left(z_{1}\right) f_{1}\left(z_{1}\right)=0, \quad f_{1}\left(z_{1}, 0\right)=1,
$$

has a unique solution satisfying

$$
0\left(\frac{f_{1}\left(z_{1}, n\right)}{f_{1}\left(z_{1}, n-1\right)}\right) \underset{n \rightarrow \pm \infty}{=} z_{1}^{ \pm 1}
$$

implying $f_{1}\left(z_{1}\right) \in l^{2}(\mathbb{Z})$. Next one defines the subcritical operator

$$
\begin{aligned}
& H_{1}^{0}\left(z_{1}, z_{2}\right):=H_{1}\left(z_{1}\right)-\Omega_{1}+\Omega_{2}, \\
& \Omega_{2}>\Omega_{1}>1, \Omega_{j}=\left(z_{j}+z_{j}^{-1}\right) / 2,0<z_{j}<1, j=1,2,
\end{aligned}
$$

with spectrum

$$
\sigma\left(H_{1}^{0}\left(z_{1}, z_{2}\right)\right)=\left\{\Omega_{2}-\Omega_{1}\right\} \cup\left[-1+\Omega_{2}, 1+\Omega_{2}\right]
$$

and repeats the above procedure. We summarize the result in

Theorem 3.1. The above construction yields operators

$$
\begin{aligned}
& H_{N}\left(z_{1}, \ldots, z_{N}\right)=A\left(z_{1}, \ldots, z_{N}\right) A\left(z_{1}, \ldots, z_{N}\right)^{*} \\
& \quad=a_{N}\left(z_{1}, \ldots, z_{N}\right) S^{+}+a_{N}^{-}\left(z_{1}, \ldots, z_{N}\right) S^{-}-b_{N}\left(z_{1}, \ldots, z_{N}\right),
\end{aligned}
$$

where

$$
A\left(z_{1}, \ldots, z_{N}\right)=\psi_{N}\left(z_{1}, \ldots, z_{N}\right) S^{+}+\phi_{N}\left(z_{1}, \ldots, z_{N}\right)
$$

and

$$
\begin{aligned}
a_{N}\left(z_{1}, \ldots, z_{N}, n\right) & \\
= & \frac{-\left[W\left(\rho_{1}, \ldots, \rho_{N}\right)(n+2)\right]^{1 / 2}\left[W\left(\rho_{1}, \ldots, \rho_{N}\right)(n)\right]^{1 / 2}}{2 W\left(\rho_{1}, \ldots, \rho_{N}\right)(n+1)}, \\
b_{N}\left(z_{1}, \ldots, z_{N}, n\right) & \\
= & -\frac{1}{2}\left[\frac{W\left(\rho_{1}, \ldots, \rho_{N-1}\right)(n+2) W\left(\rho_{1}, \ldots, \rho_{N}\right)(n)}{W\left(\rho_{1}, \ldots, \rho_{N-1}\right)(n+1) W\left(\rho_{1}, \ldots, \rho_{N}\right)(n+1)}\right. \\
& \left.+\frac{W\left(\rho_{1}, \ldots, \rho_{N-1}\right)(n) W\left(\rho_{1}, \ldots, \rho_{N}\right)(n+1)}{W\left(\rho_{1}, \ldots, \rho_{N-1}(n+1) W\left(\rho_{1}, \ldots, \rho_{N}\right)(n)\right.}\right], \quad n \in \mathbb{Z},
\end{aligned}
$$


(3.23)

$$
\begin{aligned}
\psi_{N} & \left(z_{1}, \ldots, z_{N}, n\right) \\
\quad= & {\left[\frac{W\left(\rho_{1}, \ldots, \rho_{N-1}\right)(n+2) W\left(\rho_{1}, \ldots, \rho_{N}\right)(n)}{2 W\left(\rho_{1}, \ldots, \rho_{N-1}\right)(n+1) W\left(\rho_{1}, \ldots, \rho_{N}\right)(n+1)}\right]^{1 / 2}, \quad n \in \mathbb{Z}, }
\end{aligned}
$$

$$
\begin{aligned}
\phi_{N}\left(z_{1}, \ldots, z_{N}, n\right) \\
\quad=-\left[\frac{W\left(\rho_{1}, \ldots, \rho_{N-1}\right)(n) W\left(\rho_{1}, \ldots, \rho_{N}\right)(n+1)}{2 W\left(\rho_{1}, \ldots, \rho_{N-1}\right)(n+1) W\left(\rho_{1}, \ldots, \rho_{N}\right)(n)}\right]^{1 / 2}, \quad n \in \mathbb{Z} .
\end{aligned}
$$

Here $W\left(\rho_{1}, \ldots, \rho_{N}\right)(n)$ is the discrete Wronskian, i.e.,

$$
W\left(\rho_{1}, \ldots, \rho_{N}\right)(n):=\operatorname{det}\left[\left(\left(S^{+}\right)^{j-1} \rho_{l}\right)(n)\right]_{j, l=1}^{N}
$$

and

$$
\rho_{j}(n):=z_{j}^{-n}+(-1)^{j-1} \mu_{j} z_{j}^{n}, \quad \mu_{j}>0,1 \leq j \leq N, n \in \mathbb{Z} .
$$

Moreover, $H_{N}\left(z_{1}, \ldots, z_{N}\right)$ is critical with spectrum

$$
\begin{aligned}
& \sigma\left(H_{N}\left(z_{1}, \ldots, z_{N}\right)\right)=\bigcup_{j=1}^{N}\left\{\Omega_{N}-\Omega_{j}\right\} \cup\left[-1+\Omega_{N}, 1+\Omega_{N}\right], \\
& \Omega_{j}=\frac{z_{j}+z_{j}^{-1}}{2}, \quad \begin{array}{r}
\Omega_{N}>\Omega_{N-1}>\cdots>\Omega_{1}>1, \\
0<z_{j}<1,1 \leq j \leq N, N \in \mathbb{N} .
\end{array}
\end{aligned}
$$

Next we briefly consider an alternative representation for $a_{N}, b_{N}$ due to [7].

Lemma 3.2. Define

$$
\begin{gathered}
B_{0}(t, n):=1, \quad(t, n) \in \mathbb{R} \times \mathbb{Z}, \\
B_{N}(t, n):=\left[\delta_{j, l}+c_{j}(t) c_{l}(t) \frac{\left(z_{j} z_{l}\right)^{n+1}}{1-z_{j} z_{l}}\right]_{j, l=1}^{N}, \\
N \in \mathbb{N},(t, n) \in \mathbb{R} \times \mathbb{Z},
\end{gathered}
$$

where

$$
\begin{array}{cc}
c_{j}(t)=c_{j}(0) e^{\beta_{j} t}, & c_{j}(0) \in \mathbb{C} \backslash\{0\}, \\
\beta_{j}=\left(z_{j}-z_{j}^{-1}\right) / 2, & 1 \leq j \leq N, t \in \mathbb{R} .
\end{array}
$$

Let

$$
\begin{aligned}
\eta_{j}(t, n):=z_{j}^{n} e^{z_{j} t+m_{j}}+(-1)^{j-1} z_{j}^{-n} e^{z_{j}^{-1} t-m_{j}} & \\
1 & \leq j \leq N,(t, n) \in \mathbb{R} \times \mathbb{Z},
\end{aligned}
$$

with

$$
c_{j}(0)^{2}=(-1)^{j} z_{j}^{N-3} \frac{\prod_{l=1}^{N}\left(z_{j} z_{l}-1\right)}{\prod_{l=1, l \neq j}^{N}\left(z_{l}-z_{j}\right)} e^{2 m_{j}}, \quad m_{j} \in \mathbb{C}, 1 \leq j \leq N .
$$

Then the Wronskian

$$
W\left(\eta_{1}, \ldots, \eta_{N}\right)(t, n)=\operatorname{det}\left[\left(S^{+}\right)^{j-1} \eta_{l}(t, n)\right]=\operatorname{det}\left[\partial_{t}^{j-1} \eta_{l}(t, n)\right]
$$


satisfies

$$
\begin{aligned}
\operatorname{det} B_{N}(t, n)= & {\left[\prod_{j=1}^{N}(-1)^{j-1} z_{j}^{N+n} e^{-z_{j}^{-1} t+m_{j}}\right]\left[\prod_{\substack{j, l=1 \\
j \neq l}}^{N}\left(z_{j}^{-1}-z_{l}^{-1}\right)\right]^{-1} } \\
& \cdot W\left(\eta_{1}, \ldots, \eta_{N}\right)(t, n), \quad(t, n) \in \mathbb{R} \times \mathbb{Z},
\end{aligned}
$$

and hence

$$
\begin{aligned}
\partial_{t} \ln \operatorname{det} B_{N}(t, n)=\partial_{t} \ln W\left(\eta_{1}, \ldots, \eta_{n}\right)(t, n)-\sum_{j=1}^{N} z_{j}^{-1}, & \\
& (t, n) \in \mathbb{R} \times \mathbb{Z}, N \in \mathbb{N} .
\end{aligned}
$$

Proof. Following $[8,10]$ one defines the $N \times N$ matrices

$$
\begin{aligned}
& P_{1}:=\left[\delta_{j, l} p_{j}\right], \quad P_{2}(t):=\left[\delta_{j, l} q_{j}(t)\right], \quad A=\left[\left(1-z_{j} z_{l}\right)^{-1}\right], \\
& D_{1}(t):=\left[\delta_{j, l} d_{j}(t)\right], \quad D_{2}:=\left[\delta_{j, l} z_{j}^{n+1}\right] \text {, } \\
& U(t):=\left[\delta_{j, l} c_{j}(t)\right], \quad V:=\left[z_{l}^{-(j-1)}\right], \quad W(t):=\left[\tau_{l}(t) z_{l}^{j-1}\right], \quad t \in \mathbb{R},
\end{aligned}
$$

where

$$
\begin{aligned}
p_{j} & := \begin{cases}1, & N=1, \\
z_{j}^{N-1} \prod_{\substack{l=1 \\
l \neq j}}^{N}\left(z_{l}-z_{j}\right)^{-1}, & N \geq 2,\end{cases} \\
q_{j}(t) & :=-z_{j}^{-1} e^{z_{j} t+m j} \prod_{l=1}^{N}\left(z_{l} z_{j}-1\right), \\
d_{j}(t): & :(-1)^{j-1} z_{j}^{n} e^{-z_{j}^{-1} t+m_{j}}, \\
\tau_{j}(t): & : z_{j}^{-1} e^{z_{j} t+m_{j}}, \quad 1 \leq j \leq N, \quad t \in \mathbb{R} .
\end{aligned}
$$

A tedious calculation then shows that

$$
\begin{array}{r}
U(t) B_{N}(t, n) U(t)^{-1}=P_{2}(t) D_{1}(t) V^{-1}\left[V D_{1}(t)^{-1}+W(t) D_{2}\right] P_{2}(t)^{-1}, \\
t \in \mathbb{R},
\end{array}
$$

and

$$
\begin{array}{r}
\operatorname{det}\left[V D_{1}(t)^{-1}+W(t) D_{2}\right](t, n)=W\left(\eta_{1}, \ldots, \eta_{N}\right)(t, n), \\
(t, n) \in \mathbb{R} \times \mathbb{Z},
\end{array}
$$

proving (3.32).

Inserting the $t$-dependence (3.29) into (3.22) applying Lemma 3.2 then yields 
the $N$-soliton solutions $a_{N}, b_{N}$ of the Toda lattice in the form

$$
\begin{array}{r}
a_{N}\left(z_{1}, \ldots, z_{N}, t, n\right)=-\frac{\left[\operatorname{det} B_{N}(t, n+2)\right]^{1 / 2}\left[\operatorname{det} B_{N}(t, n)\right]^{1 / 2}}{2 \operatorname{det} B_{N}(t, n+1)} \\
=-\frac{\left[W\left(\eta_{1}, \ldots, \eta_{N}\right)(t, n+2)\right]^{1 / 2}\left[W\left(\eta_{1}, \ldots, \eta_{N}\right)(t, n)\right]^{1 / 2}}{2 W\left(\eta_{1}, \ldots, \eta_{n}\right)(t, n+1)}, \\
b_{N}\left(z_{1}, \ldots, z_{N}, t, n\right)=-\frac{1}{2}\left[\frac{z_{N} \operatorname{det} B_{N-1}(t, n+2) \operatorname{det} B_{N}(t, n)}{\operatorname{det} B_{N-1}(t, n+1) \operatorname{det} B_{N}(t, n+1)}\right. \\
\left.+\frac{\operatorname{det} B_{N-1}(t, n) \operatorname{det} B_{N}(t, n+1)}{z_{N} \operatorname{det} B_{N-1}(t, n+1) \operatorname{det} B_{N}(t, n)}\right] \\
=-\frac{1}{2}\left[\frac{W\left(\eta_{1}, \ldots, \eta_{N-1}\right)(t, n+2) W\left(\eta_{1}, \ldots, \eta_{N}\right)(t, n)}{W\left(\eta_{1}, \ldots, \eta_{N-1}\right)(t, n+1) W\left(\eta_{1}, \ldots, \eta_{N}\right)(t, n+1)}\right. \\
\left.+\frac{W\left(\eta_{1}, \ldots, \eta_{N-1}\right)(t, n) W\left(\eta_{1}, \ldots, \eta_{N}\right)(t, n+1)}{W\left(\eta_{1}, \ldots, \eta_{N-1}\right)(t, n+1) W\left(\eta_{1}, \ldots, \eta_{N}\right)(t, n)}\right], \\
\operatorname{TL}\left(a_{N}, b_{N}\right)=0, \quad N \in \mathbb{N} . \quad(t, n) \in \mathbb{R} \times \mathbb{Z},
\end{array}
$$

Uniqueness of the inverse scattering method for the Toda lattice implies that one may alternatively write $b_{N}$ as

$$
\begin{array}{r}
b_{N}\left(z_{1}, \ldots, z_{N}, t, n\right)=-\frac{1}{2}\left(z_{N}+z_{N}^{-1}\right)+\partial_{t} \ln \left[\frac{\operatorname{det} B_{N}(t, n+1)}{\operatorname{det} B_{N}(t, n)}\right] \\
=-\frac{1}{2}\left(z_{N}+z_{N}^{-1}\right)+\partial_{t} \ln \left[\frac{W\left(\eta_{1}, \ldots, \eta_{N}\right)(t, n+1)}{W\left(\eta_{1}, \ldots, \eta_{N}\right)(t, n)}\right], \\
(t, n) \in \mathbb{R} \times \mathbb{Z}
\end{array}
$$

(the constant $-\left(z_{N}+z_{N}^{-1}\right) / 2$ being determined by the asymptotic behavior as $|n| \rightarrow \infty$.) Theorem 2.9 now yields (identifying $H_{1}(t)=H_{N}\left(z_{1}, \ldots, z_{N}, t\right)$, $a_{1}(t)=a_{N}\left(z_{1}, \ldots, z_{N}, t\right), b_{1}(t)=b_{N}\left(z_{1}, \ldots, z_{N}, t\right)$, etc. $)$

Theorem 3.3. Since $H_{N}\left(z_{1}, \ldots, z_{N}, t\right)$ is critical, the $(2 N-1)$-soliton solutions of the Kac-van Moerbeke lattice are given by

$$
\begin{aligned}
& \alpha_{2 N-1}\left(z_{1}, \ldots, z_{N}, t, n\right) \\
& =\left\{\begin{array}{ll}
\phi_{N}\left(z_{1}, \ldots, z_{N}, t, m\right), & n=2 m, \\
\psi_{N}\left(z_{1}, \ldots, z_{N}, t, m\right), & n=2 m+1,
\end{array} \quad(t, m) \in \mathbb{R} \times \mathbb{Z},\right. \\
& \operatorname{KM}\left(\alpha_{2 N-1}\right)=0, \quad N \in \mathbb{N},
\end{aligned}
$$

where

$$
\begin{array}{r}
\phi_{N}\left(z_{1}, \ldots, z_{N}, t, m\right) \\
=-\left[\frac{W\left(\eta_{1}, \ldots, \eta_{N-1}\right)(t, n) W\left(\eta_{1}, \ldots, \eta_{N}\right)(t, n+1)}{2 W\left(\eta_{1}, \ldots, \eta_{N-1}\right)(t, n+1) W\left(\eta_{1}, \ldots, \eta_{N}\right)(t, n)}\right]^{1 / 2}, \\
(t, m) \in \mathbb{R} \times \mathbb{Z}, \\
\psi_{N}\left(z_{1}, \ldots, z_{N}, t, m\right) \\
=\left[\frac{W\left(\eta_{1}, \ldots, \eta_{N-1}\right)(t, n+2) W\left(\eta_{1}, \ldots, \eta_{N}\right)(t, n)}{2 W\left(\eta_{1}, \ldots, \eta_{N-1}\right)(t, n+1) W\left(\eta_{1}, \ldots, \eta_{N}\right)(t, n+1)}\right]^{1 / 2}, \\
(t, m) \in \mathbb{R} \times \mathbb{Z},
\end{array}
$$


and $\eta_{j}(t, n)$ are given by (3.30). The corresponding Dirac operator $Q_{2 N-1}(t)$ (see (2.13)) then has spectrum

$$
\begin{aligned}
\sigma\left(Q_{2 N-1}(t)\right)= & \bigcup_{j=1}^{N}\left\{ \pm\left|\Omega_{N}-\Omega_{j}\right|^{1 / 2}\right\} \cup\left[-\left|1+\Omega_{N}\right|^{1 / 2},-\left|-1+\Omega_{N}\right|^{1 / 2}\right] \\
& \cup\left[\left|-1+\Omega_{N}\right|^{1 / 2},\left|1+\Omega_{N}\right|^{1 / 2}\right], \quad t \in \mathbb{R}, N \in \mathbb{N} .
\end{aligned}
$$

Example 3.4. In the critical case the 1-soliton solution is given by

$$
\begin{aligned}
\alpha_{1}\left(z_{1}, t, n\right)= \begin{cases}-\left[\frac{1+c_{1}(t)^{2} z_{1}^{2 m+2}\left(1-z_{1}^{2}\right)^{-1}}{2 z_{1}\left[1+c_{1}(t)^{2} z_{1}^{2 m}\left(1-z_{1}^{2}\right)^{-1}\right]}\right]^{1 / 2}, & n=2 m, \\
{\left[\frac{z_{1}\left[1+c_{1}(t)^{2} z_{1}^{2 m}\left(1-z_{1}^{2}\right)^{-1}\right]}{2\left[1+c_{1}(t)^{2} z_{1}^{2 m+2}\left(1-z_{1}^{2}\right)^{-1}\right]}\right]^{1 / 2},} & n=2 m+1,\end{cases} \\
c_{1}(t)=c_{1}(0) e^{\left(z_{1}-z_{1}^{-1}\right) t / 2}, \quad(t, m) \in \mathbb{R} \times \mathbb{Z},
\end{aligned}
$$

$$
\mathrm{KM}\left(\alpha_{1}\right)=0 .
$$

Remark 3.5. In the subcritical case one can obtain the Kac-van Moerbeke $(2 \mathrm{~N}-$ 2)-soliton solutions by "turning off" the eigenvalue at zero of $H_{N}\left(z_{1}, \ldots, z_{N}, t\right)$ (rendering it subcritical). This is most conveniently accomplished by using the formulas expressed in det $B_{N}(t, n)$ letting $c_{N}(0) \rightarrow 0$. One then obtains

$$
\begin{gathered}
\alpha_{2 N-2}\left(z_{1}, \ldots, z_{N}, t, n\right) \\
=\left\{\begin{array}{ll}
\tilde{\phi}_{N}\left(z_{1}, \ldots, z_{N}, t, m\right), & n=2 m, \\
\tilde{\psi}_{N}\left(z_{1}, \ldots, z_{n}, t, m\right), & n=2 m+1,
\end{array} \quad(t, m) \in \mathbb{R} \times \mathbb{Z},\right. \\
\operatorname{KM}\left(\alpha_{2 N-2}\right)=0, \quad N \in \mathbb{N},
\end{gathered}
$$

where

$$
\begin{array}{r}
\tilde{\phi}_{N}\left(z_{1}, \ldots, z_{N}, t, m\right)=-\left[\frac{\operatorname{det} B_{N-1}(t, m) \operatorname{det} \tilde{B}_{N-1}(t, m+1)}{2 z_{N} \operatorname{det} B_{N-1}(t, m+1) \operatorname{det} \tilde{B}_{N-1}(t, m)}\right]^{1 / 2} \\
=-\left[\frac{W\left(\eta_{1}, \ldots, \eta_{n-1}\right)(t, m) W\left(\tilde{\eta}_{1}, \ldots, \tilde{\eta}_{N-1}\right)(t, m+1)}{2 z_{N} W\left(\eta_{1}, \ldots, \eta_{N-1}\right)(t, m+1) W\left(\tilde{\eta}_{1}, \ldots, \tilde{\eta}_{N-1}\right)(t, m)}\right]^{1 / 2}, \\
(t, m) \in \mathbb{R} \times \mathbb{Z},
\end{array}
$$

$$
\begin{gathered}
\tilde{\psi}_{N}\left(z_{1}, \ldots, z_{N}, t, m\right)=\left[\frac{z_{N} \operatorname{det} B_{N-1}(t, m+2) \operatorname{det} \tilde{B}_{N-1}(t, m)}{2 \operatorname{det} B_{N-1}(t, m+1) \operatorname{det} \tilde{B}_{N-1}(t, m+1)}\right]^{1 / 2} \\
=\left[\frac{z_{N} W\left(\eta_{1}, \ldots, \eta_{N-1}\right)(t, m) W\left(\tilde{\eta}_{1}, \ldots, \tilde{\eta}_{N-1}\right)(t, m)}{2 W\left(\eta_{1}, \ldots, \eta_{N-1}\right)(t, m+1) W\left(\tilde{\eta}_{1}, \ldots, \tilde{\eta}_{N-1}\right)(t, m+1)}\right]^{1 / 2}, \\
(t, m) \in \mathbb{R} \times \mathbb{Z} .
\end{gathered}
$$


Here $\tilde{B}_{N-1}(t, n)$ is the matrix

(3.52)

$$
\begin{aligned}
\tilde{B}_{0}(t, n) & :=1, \quad(t, n) \in \mathbb{R} \times \mathbb{Z}, \\
\tilde{B}_{N-1}(t, n) & :=\left[\delta_{j, l}+\frac{\tilde{c}_{j}(t) \tilde{c}_{l}(t)}{1-z_{j} z_{l}}\left(z_{j} z_{l}\right)^{n+1}\right]_{j, l=1}^{N-1}, \quad N \geq 2, \quad(t, n) \in \mathbb{R} \times \mathbb{Z},
\end{aligned}
$$

with

$$
\begin{gathered}
\tilde{c}_{j}(t)=\tilde{c}_{j}(0) e^{\left(z_{j}-z_{j}^{-1}\right) t / 2}, \quad \tilde{c}_{j}(0)^{2}=z_{j} \frac{z_{j} z_{N}-1}{z_{N}-z_{j}} c_{j}(0)^{2}, \\
j=1, \ldots, N-1, t \in \mathbb{R}, \\
\tilde{\eta}_{j}(t, n)=z_{j}^{n} e^{z_{j} t+\tilde{m}_{j}}+(-1)^{j-1} z_{j}^{-n} e^{z_{j}^{-1} t-\tilde{m}_{j}}, \\
j=1, \ldots, N-1,(t, n) \in \mathbb{R} \times \mathbb{Z}, \\
\tilde{m}_{j}=m_{j}+\frac{1}{2} \ln \left[\frac{\left(z_{j} z_{N-1}\right) z_{j}}{z_{N}-z_{j}}\right], \quad j=1, \ldots, N-1 .
\end{gathered}
$$

Remark 3.6. The approach presented in this section relies on "single-commutation" [4] and therefore introduces all eigenvalues below the essential spectrum. This limitation can easily be removed by using "double-commutation" instead [30].

Remark 3.7. The first one to give the $N$-soliton solution of the Toda lattice appears to be Hirota [18]. The Toda $N$-soliton solutions expressed in terms of $\operatorname{det} B(t, n)$ are due to Flaschka [7] (see also [29]). Marchenko [22] gives the $2 N$ soliton solutions for both the TL and the KM-systems in terms of Wronskians in the subcritical case using an entirely different approach from the one advocated here. Although he does not list the soliton formulas corresponding to the critical case, they can be obtained from his expressions via an appropriate limiting procedure similar to the one employed in Remark 3.5.

Acknowledgments. Two of us (F. G. and Z. Z.) would like to thank Mark Ashbaugh for stimulating discussions. F. G. and H. H. gratefully acknowledge the support from the Norwegian Research Council for Science and the Humanities (NAVF). H. H. is grateful to Professor D. Wales for his kind invitation to Caltech.

\section{APPENDIX A. ON NONNEGATIVE, SECOND ORDER, FINITE DIFFERENCE OPERATORS}

We summarize the necessary facts on positive weak solutions of nonnegative, second order, finite difference operators on $\mathbb{Z}$ needed in this manuscript. For a novel approach and detailed proofs of the topics presented below we refer to [14]. Basic results on finite difference operators can be found, e.g., in [2, 3, 15, 17].

For simplicity we shall assume hypothesis

$$
\begin{aligned}
a=\{a(n)\}_{n \in \mathbb{Z}} \in l^{\infty}(\mathbb{Z}), \quad b= & \{b(n)\}_{n \in \mathbb{Z}} \in l^{\infty}(\mathbb{Z}), \\
& a(n)<0, \quad b(n)<0, n \in \mathbb{Z} .
\end{aligned}
$$


throughout this appendix (the assumption $a, b \in l^{\infty}(\mathbb{Z})$ is removed in [14]) and define the operator

$$
H=a S^{+}+a^{-} S^{-}-b
$$

on $l^{2}(\mathbb{Z})$. Associated with $H$ we introduce the matrices $M_{m, n}$ defined by (A.2)

$$
M_{m, n}:=\left(\begin{array}{cccccc}
-b(m) & a(m) & 0 & \ldots & & 0 \\
a(m) & -b(m+1) & a(m+1) & & & \vdots \\
0 & & & & & \\
\vdots & & & & & \\
0 & \ldots & & a(n-2) & -b(n-1) & a(n-1) \\
0 & & 0 & a(n-1) & -b(n)
\end{array}\right), \quad m<n,
$$

and the determinants

$$
d_{m, n}:=\operatorname{det}\left[M_{m, n}\right], \quad m<n .
$$

Lemma A.2. Assume (H.A.1).

(i) Let $m<n$. Then $M_{m, n} \geq 0$ implies $d_{m, n-1}>0$.

(ii) $H \geq 0$ iff $d_{m, n}>0$ for any $m<n$.

(iii) Let $H \geq 0$. Then for all $m<k<n$,

$$
\begin{aligned}
& -b(k)^{-1}<d_{k+1, n}\left(d_{k, n}\right)^{-1}<-b(k-1) a(k-1)^{-2}, \\
& -b(k)^{-1}<d_{m, k-1}\left(d_{m, k}\right)^{-1}<-b(k+1) a(k)^{-2} .
\end{aligned}
$$

(iv) For any $k \in \mathbb{Z}$, the map

$$
n \rightarrow d_{k+1, n}\left(d_{k, n}\right)^{-1}
$$

is increasing for $n>k$ and the map

$$
m \rightarrow d_{m, k-1}\left(d_{m, k}\right)^{-1}
$$

is decreasing for $m<k$.

Because of Lemma A.2(iv) we define in the case $H \geq 0$

(A.8) $\quad c(k)_{+}:=\lim _{n \rightarrow \infty} d_{k+1, n}\left(d_{k, n}\right)^{-1}, \quad c(k)_{-}:=\lim _{m \rightarrow-\infty} d_{m, k-1}\left(d_{m, k}\right)^{-1}$

and the following positive functions on $\mathbb{Z} \times \mathbb{Z}$ :

$$
\begin{aligned}
& \text { (A.9) } u(l, k):= \begin{cases}1, & l=k, \\
{[-a(l)] \cdots[-a(k-1)] c(l+1)_{+} \cdots c(k)_{+},} & l<k, \\
{[-a(k)] \cdots[-a(l-1)] c(k)_{-} \cdots c(l-1)_{-},} & l>k,\end{cases} \\
& \text { (A.10) } u_{+}(k):= \begin{cases}1, & k=0, \\
u(0, k), & k>0, \\
u(k, 0)^{-1}, & k<0,\end{cases} \\
& \text { (A.11) } u_{-}(k):= \begin{cases}1, & k=0, \\
u(k, 0)^{-1}, & k>0, \\
u(0, k), & k<0 .\end{cases}
\end{aligned}
$$

We have 
Lemma A.3. Assume (H.A.1) and $H \geq 0$. Then

$$
\begin{gathered}
-b(k)^{-1} \leq c(k)_{+} \leq-b(k-1) a(k-1)^{-2}, \\
-b(k)^{-1} \leq c(k)_{-} \leq-b(k+1) a(k)^{-2} .
\end{gathered}
$$

(ii) $k \rightarrow u(l, k)$ is the minimal weak positive solution of $H f=0$ with $f(l)=1$ for $k \geq l$ and $k \leq l$ separately (i.e., if $v>0$ is a weak positive solution of $H v=0$ with $v(l)=1$ in $[l, \infty) \cap \mathbb{Z}$ then $v(k) \geq u(l, k)$ for all $k \geq l$ and similarly on $(-\infty, l] \cap \mathbb{Z})$.

(iii) $u_{ \pm}$are weak positive solutions of $H f=0$ on $\mathbb{Z}$.

(iv) Let $u_{j}>0, j=1,2$, be positive solutions of $H f=0$ with $u_{1}(0)=$ $u_{2}(0)$. If for some $k_{0}>0, u_{1}\left(k_{0}\right) \underset{(=)}{>} u_{2}\left(k_{0}\right)$ then $u_{1}(k) \underset{(=)}{>} u_{2}(k)$ for all $k>0$. Similarly, if for some $k_{0}<0, u_{1}\left(k_{0}\right) \geq u_{2}\left(k_{0}\right)$ then $u_{1}(k) \underset{(=)}{>} u_{2}(k)$ for all $k<0$.

(v) For any $m \leq l \leq k$ we have

$$
u(m, k)=u(m, l) u(l, k), \quad u(k, m)=u(k, l) u(l, m) .
$$

(vi) For any $l, m \in \mathbb{Z}$,

$$
u(m, l) u(l, m) \leq 1
$$

$$
\begin{aligned}
\sum_{k=n}^{\infty}[-a(k) & \left.u_{+}(k) u_{+}(k+1)\right]^{-1}=\infty \\
= & \sum_{k=n}^{-\infty}\left[-a(k) u_{-}(k) u_{-}(k+1)\right]^{-1}, \quad n \in \mathbb{Z}
\end{aligned}
$$

Remark A.4. The weak positive solutions $u_{ \pm}$are the analogs of principal solutions near $\pm \infty$ of nonoscillatory Sturm-Liouville operators on $\mathbb{R}$ (see, e.g., $[13,16])$. They are also called recessive in the literature (see, e.g., [27]). One can show [27] that if $H \geq 0$ and $u_{ \pm}>0$ are principal solutions of $H f=0$ near $\pm \infty$

$$
\begin{array}{ll}
\hat{u}_{+}(n):=u_{+}(n) \sum_{j=m}^{n-1}\left[-a(j) u_{+}(j) u_{+}(j+1)\right]^{-1}, & n \geq m+1, \\
\hat{u}_{-}(n):=u_{-}(n) \sum_{j=n}^{m}\left[-a(j) u_{-}(j) u_{-}(j+1)\right]^{-1}, & n \leq m,
\end{array}
$$

are nonprincipal (or dominant) solutions of $H f=0$. Conversely, if $\hat{u}_{ \pm}>0$ are nonprincipal solutions of $H f=0$ near $\pm \infty$ then

$$
u_{+}(n):=\hat{u}_{+}(n) \sum_{j=n}^{\infty}\left[-a(j) \hat{u}_{+}(j) \hat{u}_{+}(j+1)\right]^{-1}, \quad n \in \mathbb{Z},
$$

$$
u_{-}(n):=\hat{u}_{-}(n) \sum_{j=n-1}^{-\infty}\left[-a(j) \hat{u}_{-}(j) \hat{u}_{-}(j+1)\right]^{-1}, \quad n \in \mathbb{Z}
$$


are principal solutions of $H f=0$ near $\pm \infty$. In any case, nonprincipal solutions $\hat{u}_{ \pm}>0$ near $\pm \infty$ are characterized by [27]

$$
\sum_{j=n}^{ \pm \infty}\left[-a(n) \hat{u}_{ \pm}(j) \hat{u}_{ \pm}(j+1)\right]^{-1}<\infty, \quad n \in \mathbb{Z}
$$

We also recall

Theorem A.5. Assume (H.A.1). Then $H \geq 0$ iff there exists a weak positive solution $u>0$ of $H f=0$.

Moreover, we have

Theorem A.6 [12]. Let $H \geq 0$. Then conditions (i)-(vi) are equivalent.

(i) There exists a unique weak positive solution $u>0$ of $H f=0$ with $u(0)=1$.

(ii) $u_{+}=u_{-}$.

(iii) For some $k, l \in \mathbb{Z}, k \neq l$,

$$
u(k, l) u(l, k)=1 \text {. }
$$

(iv) For any $k, l \in \mathbb{Z}$,

$$
u(k, l) u(l, k)=1 \text {. }
$$

(v) For some $k \in \mathbb{Z}$,

$$
a(k)^{2} c(k)_{-} c(k+1)_{+}=1 \text {. }
$$

(vi) For any $k \in \mathbb{Z}$,

$$
a(k)^{2} c(k)_{-} c(k+1)_{+}=1 .
$$

Because of Theorems A.5 and A.6 we introduce in analogy to Sturm-Liouville and general Schrödinger-type operators $[13,25,28]$ the definition

Definition A.7. Let $H \geq 0$. Then $H$ is called critical iff $H$ satisfies one of the conditions (i) $-(\mathrm{vi})$ in Theorem A.6. Otherwise $H$ is called subcritical.

In particular, $H \geq 0$ is critical (or subcritical) iff $H f=0$ has a unique (or two linearly independent) positive solution(s) up to multiples of constants.

In the following we write $H(a, b)$ instead of $H$ in order to exhibit the $(a, b)$-dependence of $H$.

Theorem A.8 [14]. Let $H(a, b) \geq 0$. Then the following conditions (i)-(v) are equivalent.

(i) $H(a, b)$ is critical.

(ii) For some $k \in \mathbb{Z}$ and any $\epsilon>0, H\left(a-\epsilon \delta_{k}, b\right) \geq 0$.

(iii) For any $k \in \mathbb{Z}$ and any $\epsilon>0, H\left(a-\epsilon \delta_{k}, b\right) \geq 0$.

(iv) For some $k \in \mathbb{Z}$ and any $\epsilon>0, H\left(a, b+\epsilon \delta_{k}\right) \geq 0$.

(v) For any $k \in \mathbb{Z}$ and any $\epsilon>0, H\left(a, b+\epsilon \delta_{k}\right) \geq 0$, where

$$
\delta_{k}(n)= \begin{cases}1, & k=n, \\ 0, & k \neq n .\end{cases}
$$

Let $l_{0}(\mathbb{Z})$ denote the set of complex-valued sequences $g=\{g(n)\}_{n \in \mathbf{Z}}$ with only finitely many $g(n) \neq 0$. Then we get

Corollary A.9. Let $H(a, b) \geq 0$. Then $H(a, b)$ is subcritical iff for any real valued $f, g \in l_{0}(\mathbb{Z})$ there exists an $\epsilon>0$ such that $H(a+\epsilon f, b+\epsilon g) \geq 0$. 


\section{REFERENCES}

1. M. Adler, On the Bäcklund transformation for the Gel'fand-Dickey equations, Comm. Math. Phys. 80 (1981), 517-527.

2. F. V. Atkinson, Discrete and continuous boundary problems, Academic Press, New York, 1964.

3. Ju. M. Berezanskii, Expansions in eigenfunctions of selfadjoint operators, Transl. Math. Monographs, vol. 17, Amer. Math. Soc., Providence, R.I., 1968.

4. P. Deift, Applications of a commutation formula, Duke Math. J. 45 (1976), 267-310.

5. P. Deift, L.C. Li, and C. Tomei, Toda flows with infinitely many variables, J. Funct. Anal. 64 (1985), 358-402.

6. P. Deift and E. Trubowitz, Inverse scattering on the line, Comm. Pure Appl. Math. 32 (1979), 121-251.

7. H. Flaschka, On the Toda lattice. II, Progr. Theoret. Phys. 51 (1974), 703-716.

8. N. C. Freeman and J. J. C. Nimmo, Soliton solutions of the Korteweg-de Vries and the Kadomtsev-Petviashvili equations: the Wronskian technique, Proc. Roy. Soc. London Ser. A 389 (1983), 319-329.

9. F. Gesztesy, H. Holden, E. Saab, and B. Simon, Explicit construction of solutions of the modified Kadomtsev-Petviashvili equation, J. Funct. Anal. 98 (1991), 211-228.

10. F. Gesztesy and W. Schweiger, Rational KP- and mKP-solutions in Wronskian form, Rep. Math. Phys. 30 (1991), 205-222.

11. F. Gesztesy, W. Schweiger, and B. Simon, Commutation methods applied to the mKdVequation, Trans. Amer. Math. Soc. 324 (1991), 465-525.

12. F. Gesztesy and B. Simon, Constructing solutions of the $m K d V$-equation, J. Funct. Anal. 89 (1990), 53-60.

13. F. Gesztesy and Z. Zhao, On critical and subcritical Sturm-Liouville operators, J. Funct. Anal. 98 (1991), 311-345.

14. __ On critical and subcritical Jacobi operators, J. Differential Equations (to appear).

15. I. M. Glazman, Direct methods of qualitative spectral analysis of singular differential operators, Israel Program for Scientific Translations, Jerusalem, 1965.

16. P. Hartman, Ordinary differential equations, 2nd ed., Birkhäuser, Boston, Mass., 1982.

17. D. B. Hinton and R. T. Lewis, Spectral analysis of second order difference equations, J. Math. Anal. Appl. 63 (1978), 421-438.

18. R. Hirota, Exact $N$-soliton solution of a nonlinear lumped network equation, J. Phys. Soc. Japan 35 (1973), 286-288.

19. M. Kac and P. van Moerbeke, On an explicitly soluble system of nonlinear differential equations related to certain Toda lattices, Adv. Math. 16 (1975), 160-169.

20. __ On some periodic Toda lattices, Proc. Nat. Acad. Sci. U.S.A. 72 (1975), 1627-1629.

21. S. V. Manakov, Complete integrability and stochastization of discrete dynamical systems, Soviet. Phys. JETP 40 (1975), 269-274.

22. V. A. Marchenko, Nonlinear equations and operator algebras, Reidel, Dordrecht, 1988.

23. R. M. Miura, Korteweg-de Vries equation and generalization. I. A remarkable explicit nonlinear transformation, J. Math. Phys. 9 (1968), 1202-1204.

24. J. Moser, Three integrable Hamiltonian systems connected with isospectral deformations, Adv. Math. 16 (1975), 197-220.

25. M. Murata, Structure of positive solutions to $(-\Delta+V) u=0$ in $\mathbb{R}^{n}$, Duke Math. J. 53 (1986), 869-943.

26. S. Novikov, S. V. Manakov, L. P. Pitaevskii and V. E. Zakharov, Theory of solitons, Consultants Bureau, New York, 1984.

27. W. T. Patula, Growth and oscillation properties of second order linear difference equations, SIAM J. Math. Anal. 10 (1979), 55-61. 
28. B. Simon, Large time behavior of the $L^{p}$ norm of Schrödinger semigroups, J. Funct. Anal. 40 (1981), 66-83.

29. M. Toda, Theory of nonlinear lattices, 2nd enlarged ed., Springer-Verlag, Berlin, 1989.

30. S. Venakides, P. Deift, and R. Oba, The Toda shock problem, preprint, New York University, 1990.

31. S. Yamazaki, On the system of nonlinear differential equations $y_{k}=y_{k}\left(y_{k+1}-y_{k-1}\right), \mathbf{J}$. Phys. A 20 (1987), 6237-6241.

32. V. E. Zakharov, S. L. Musher, and A. M. Rubenchik, Nonlinear state of parametric wave excitation in a plasma, JETP Lett. 19 (1974), 151-152.

(F. Gesztesy and Z. Zhao) Department of Mathematics, University of Missouri, Columbia, Missouri 65211

E-mail address: mathfg@mizzou1.missouri.edu

E-mail address: mathzz@mizzou1.missouri.edu

(H. Holden) Division of Mathematical Sciences, The Norwegian Institute of TechnologY, THE University OF TRONDHEIM, N-7034 TRONDHEIM, NoRWAY

E-mail address: holden@imf.unit.no

(B. Simon) Division of Physics, Mathematics and Astronomy, California Institute of Technology, Pasadena, California 91125 